NASA Technical Memorandum 105214

AIAA- $-92-1293$

\title{
MisTec: A Software Application for Supporting Space Exploration Scenario Options and Technology Development Analysis and Planning
}

Gary A.P. Horsham

Lewis Research Center

Cleveland, Ohio

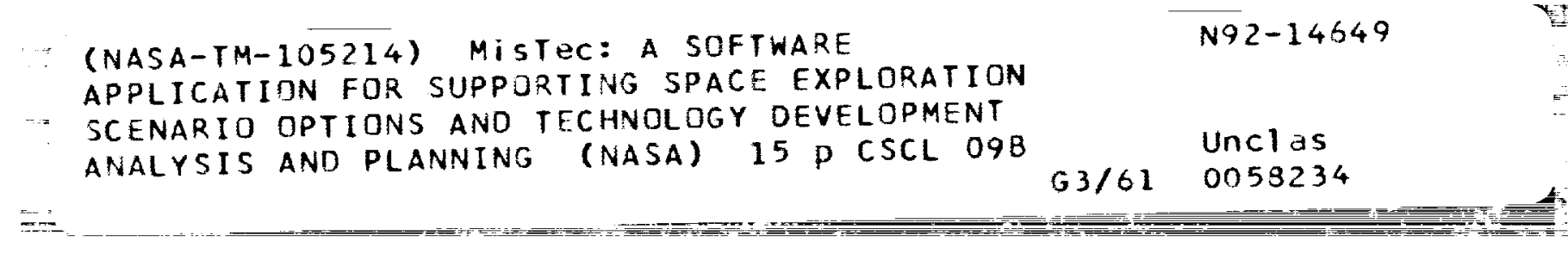

Prepared for the

Space Programs and Technologies Conference

sponsored by the American Institute of Aeronautics and Astronautics

Huntsville, Alabama, March 24-27, 1992

\section{N/Sก}




\title{
MisTec: A SOFTWARE APPLICATION FOR SUPPORTING SPACE EXPLORATION SCENARIO OPTIONS AND TECHNOLOGY DEVELOPMENT
}

\section{ANALYSIS AND PLANNING}

\author{
Gary A.P. Horsham \\ National Aeronautics and Space Administration \\ Lewis Research Center \\ Cleveland, Ohio 44135
}

\begin{abstract}
This paper presents the structure and composition of a new, emerging software application, which models and analyzes space exploration scenario options for feasibility based on technology development projections.

The software application consists of four main components: a scenario generator for designing and inputting scenario options and constraints; a processor which performs algorithmic coupling and options analyses of mission activity requirements and technology capabilities; a results display which graphically and textually shows coupling and options analysis results; and, a data/knowledge base which contains information on a variety of mission activities and (power and propulsion) technology system capabilities.
\end{abstract}

The general long-range study process used by NASA to support recent studies is briefly introduced to provide the primary basis for comparison for discussing the potential advantages to be gained from developing and applying this kind of application. The paper presents a hypothetical example of a scenario option to facilitate the best conceptual understanding of what the application is, how it works, or the operating methodology, and when it might be applied.

\section{Introduction/Background}

The NASA process for studying top-level future space exploration scenarios integrates information from many areas of expertise policy analysis, scenario strategy, and, missions and technology planning. This process is largely unstructured. Essentially, it entails the interaction (or coupling) of assumptions about future scenario (mission activity) requirements and system (technology) capabilities. If a software application can be developed to model these interactions, exploration scenario options analysis may be accomplished more rapidly and objectively (for example, the four options which resulted from the "Report of the Synthesis Group on the Space Exploration Initiative - America at the Threshold, America's Space Exploration Initiative - May, 1991," may be analyzed more thoroughly, rapidly and systematically). The software application, called "MisTec," is under development to satisfy the need for an analytical tool to support top-level long-range/strategic options assessments, and improve process efficiency.

The MisTec project promises to result in an unbiased scenario options analysis and technology development planning, software application. It should provide a software environment for input and analysis of expert judgements about projected mission activity technology requirements, and also, technology development capabilities and readiness projections. The application's four design, development and operational objectives are as follows:

- Computerize the assessment of technology requirements and availability projections for exploration scenario options.

- Provide an evolutionary software capability which is easy to modify and expand to meet changing/increasing needs. 
- Improve the capability to analyze unbiased scenario options and plan technology development programs.

- Decrease the time required per iteration to perform top-level investigations of multiple exploration scenario options.

A working prototype under contract NAS3-25266 was developed during fiscal year (FY) 1989 to demonstrate concept feasibility (Phase I). Following this, a "soft" working version or semi-operational capability (Phase II) was developed during FY 1990. The design and development approach has been based on a hybrid activity scheduling problem classification and objects (or trees) data representation. The chosen solution technique was hybrid heuristic, which was implemented using the Trees-pls programming language. A workstation class minicomputer was used to provide the necessary hardware environment.

This paper describes the structure and composition of the semi-operational (Phase II) capability and offers much insight into the application's long-term, potential applicability.

\section{Exploration Study Process}

The typical process or methodology used by NASA (Fig. 1 without shading) to identify and analyze exploration scenario options is described in Appendix A. The process is iterative and normally consists of three phases: conceptual, system engineering, and synthesis.

To exercise its full capabilities requires that MisTec be applied in the shaded area shown in Fig. 1. This represents greater computerized support as a substitute for some resource intensive activities. Acceleration or improved process iterative/cycle efficiency (reduced time) is achieved if applied in this manner. The system would serve as a data/ knowledge base repository and tracking mechanism for output traceability, as well as an integrating analytical tool.

\section{System Definition and Function}

General Input/Output

The MisTec system is designed to contribute to more rapid investigations of the interaction between exploration scenario options specifications and projected technology development timelines. The system receives and analyzes scenario options and data (Fig. 2) in a continuous feedback loop. (The data/knowledge base must be serviced initially to facilitate system start-up.) Technology requirements imposed by mission activities are assessed during analysis. The output then indicates the potential for projected technology capabilities to satisfy those requirements. Prior to modeling and further definition, a typical scenario option for analysis may be considered in the following manner:

"What power and/or propulsion technologies might be available to satisfy the power and propulsion requirements of exploration scenario $\mathrm{X}$, unfolding between 1995 and 2020, in the Earthmoon-Mars domain, according to certain constraints?"

The MisTec system model consists of four main components (Fig. 3):

- Scenario Generator - A graphical userinterface for modeling scenario options. (Appendix B.)

- Processor - An engine which contains coupling, options analysis and scheduling algorithms. (Following section.)

- Data/Knowledge Base - Data tree structures for constructing and storing timeline profiles of missions and technology requirements, capabilities and readiness. (Appendix C.)

- Results Display - A graphical interface for presenting coupling and analysis results. (Fig. 10.) 


\section{Operation and Example Application}

The input/output operating procedure (Fig. 4) consists of a series of manual and automatic actions. At Steps 1 and 2, the user manually designs an exploration scenario and specifies appropriate objectives and attributes, and decides what inheritance and synergy factors (see the following section), and technology availability constraints, will be used to produce the results. These first two steps represent the system's input. At Step 3, mission activities based on the objectives and attributes specified in Step 2 are automatically selected from the data base (not shown). At Step 4, alternative technologies based on the attributes specified in Step 2, and the activities selected in Step 3, are automatically selected from the data base. Finally, these alternative technologies are analyzed, ranked and scheduled in Step 5. Steps 3 to 5 are essentially output.

An example scenario option with temporal and space relationships between the Earth, Moon and Mars (Fig. 5) is considered for analysis. It consists of three planetary surface nodes, two low Earth orbit nodes, including one low lunar and one low Mars orbit node. Each node is defined by different objectives (construction/assembly, transportation, etc.) and occur at different points in time. Arrows indicate transportation between surface and orbit and orbital nodes.

The MisTec representation or modeling of this scenario is accomplished using a network (Fig. 6). Each node, whether planetary surface or orbital, is portrayed as a circle. $\mathbf{A}$ transportation segment (or arc) is a straight line connecting two nodes.

The low Mars orbit (LMO) node (highlighted in Fig. 6) is used to illustrate how mission activities and alternative technologies are selected or coupled. The scenario objective, "exploration/prospecting," (Fig. 7) drives this process. The corresponding objective heading is located in the data base, and mission activities are searched until matching attributes are found. If this is successful mission activities are assigned to the node as shown in the last box. This example results in two activities (determination of regolith composition, and study of climactic interactions) assigned to the LMO node.

Before technology alternatives are selected, the nodal (power) technology requirement(s) must be determined from an analysis of the requirement(s) from each activity assigned to the node. This is drawn from the mission activities data base requirement timeline profiles (not shown in figure).

The LMO node power requirement is $100 \mathrm{~kW}$ (Fig. 8), which is the power required to serve the larger need (this assumes that if the larger/peak need can be satisfied, then so can the smaller/average). A nuclear thermoelectric power technology is selected as an alternative from the technology data base. Technology capability and readiness timeline profiles are analyzed for each possible alternative. The final selection(s) is based on the matching attributes between the technology and the nodal objective, and a $200 \mathrm{~kW}$ power production capability greater than the $100 \mathrm{~kW}$ requirement.

After the technology to node coupling process is complete the results are displayed (Fig. 9). The scenario portrays alternative technologies assigned to nodes and arcs. This represents one of the first true output products of the MisTec system. Two technology alternatives are shown for the LMO node. This means that both technologies passed the nodal requirement and attribute matching test.

The two most significant output products are the options/alternatives rankings, and, the readiness critical path (Fig. 10). Numerical rankings are achieved by calculating and comparing descriptive utility metrics for each of the possible technology combinations. Each of the ranked technology combinations can then be individually displayed to show the readiness/development milestones. 
As illustrated, readiness milestones for each relevant parameter are displayed relative to temporal location of nodes and arcs (Fig. 6). This defines a feasible scenario option result.

\section{Options Analysis Formulations}

In order to rank the numerous alternative technology combination solution sets which may emerge from the technology to node coupling process, the average utility is calculated. Utility is defined as a descriptive measure of the degree of importance or the relative expected value of a technology (or node, or arc). A ranking of each alternative technology combination solution set is achieved by comparing combined utility quantities. The utility function which determines the combined utility for intradisciplinary (e.g., power) coupling of an alternative technology combination solution set is defined by;

$$
\mathrm{Uc1}=\mathrm{f}[\mathrm{Ut}(\mathrm{So}), \quad \mathrm{It}]
$$

where Uc1 represents the combined utility for intradiscipline coupling, Ut represents the utility of each technology in the alternative technology combination solution set, So represents exploration scenario objectives, and It represents an inheritance factor which relates potential/possible beneficiary technology development advantages (already available technological knowhow, development capitalization reductions, etc.) which may be gained by one technology development process from another. (Appendix D1 shows the present working formulation of this combined utility function.)

If more than one technology discipline is used in the assessment of a scenario option, the system will calculate the combined utility for multidisciplinary (e.g., propulsion with power) coupling. The utility function for this is defined by;

$$
\mathrm{Uc2}=\mathrm{f}[\mathrm{Ut}(\mathrm{So}), \quad \mathrm{Gt}]
$$

where Uc2 represents the combined utility for multidisciplinary coupling, Ut represents the utility of each technology in the alternative technology combination solution set, So represents exploration scenario objectives, and Gt represents a synergy factor which relates the potential or possible operational complementarity of one technology to another (Appendix D2 shows the present working formulation of this combined utility function.)

Alternative combination solution sets are ranked according to the highest or maximum average combined utility. This ranking represents a descriptive or comparative range indicating the most feasible alternatives (i.e., only the comparative or relative values are meaningful not the absolute values). The most feasible alternative combination solution set defines the optimal or best exploration scenario, based on the supporting assumptions, data and information.

\section{Conclusions}

It is possible to develop a software application which operates on technology requirements, capability and readiness projections data to support strategic space exploration options analysis and long-range technology development assessments. Clearly, the components that are most critical to success are the processor (with its coupling and options analysis algorithms), and the data/knowledge base.

Achieving the goal of developing and using the application to produce optimized results is quite challenging and difficult. This is because the data/knowledge base requires subjective information which can only be gathered through an evolutionary or iterative process (i.e., interaction with the relevant expert communities). It is clear that, after the data/knowledge base has been sufficiently refined and augmented through several iterations, the application should approach a high level of credibility.

The application's design and development approach (objective driven, node and arc network scenario input display, output displays, coupling algorithms, networks and data 
trees, utility metrics, etc.) appears sound. Clearly, the developmental approach might serve as an experience block upon which more advanced variations can be built.

Further development efforts need to be directed at improving the mission activity to node and technology to node selection/ coupling process by developing better coupling models. In the near-term, additional analytical capabilities (such as cost-risk analysis) could be incorporated to supplement the utility-based methodology now being applied. The utility metrics for options analysis and ranking of coupling results also should be improved. Thorough testing and hardening of the software environment will also be necessary to ensure operational reliability. Also, modification of the software to facilitate operation on more easily accessible hardware systems needs to be considered. Finally, expansion of the application to accommodate other/additional technology disciplines besides power and propulsion will remain as a most challenging task.

\section{$\underline{\text { Appendix A* }}$}

1. Conceptual Mission Analysis and Broad Trades

During this period, mission requirements are defined that meet the exploration goals and objectives and user requirements (Fig. 1). The mission requirements specify performance parameters for systems defined by a study, identify environments in which conceptual systems must operate to meet the specified requirements, and point toward the broad trade areas. The technical options available within each trade area are analyzed for their relative benefit. These trades identify the system concept options and elements that outline a study.

\footnotetext{
*Excerpted from Exploration Studies Technical Report, Vol. 1: Mission and Integrated Systems. NASA Office of Exploration, FY 1989 Annual Report.
}

\section{System Engineering}

This period encompasses system-level studies and syntheses using the results of the previous phase. A definition of each system emerges.

\section{Synthesis}

This period comprises a synthesis of the system-level studies, in which system requirements assumptions provide a basis for defining configuration options, and system-level trade studies identify the parametric cost, performance, and risk. The results also establish a preliminary system concept and a reference configuration that is used to refine the study through several iterations. The refined studies, associated requirements, and relative benefits become the knowledge base of exploration path sensitivities. The base is used to define the exploration initiative options, benefits, and risks (that support selection and subsequent decisions).

\section{Appendix B}

\section{Scenario Generator: Scenario Modeling}

The scenario modeling capability employs a network of nodes and arcs (Fig. B1). The software provides a pad on which any size network can be drawn. Nodes are to be interpreted as accumulation points for technology requirements near planets, or on planetary surfaces, where single or multiple mission activities may be performed (e.g., Low lunar Orbit (LLO-2005) in the year 2005). Arcs are to be interpreted as accumulation points for transportation technology requirements between two nodes (e.g., LLO2005 to LS-2009). Each node and arc is characterized by specifying an objective from a list of available (modifiable) choices. Similarly, attributes (A1, A2, etc.), qualitative values (V1, V2, etc.), and quantitative "utility" values (U1, U2, etc.) are subsequently specified for each objective (see Mars surface, MS2020, node in Fig. 1(a)). 


\section{Appendix C}

\section{Data/Knowledge Base: Structure and Composition}

The data/knowledge base contains information in data tree structures for rapid manipulation by coupling, options analysis and scheduling algorithms. Figures $\mathrm{C1}$ and C2 depict the general structure of the mission activity and technology data trees, respectively.

\section{Appendix D}

D1: The exact formulation ${ }^{* *}$ of the intradiscipline combined utility summation function is written as;

$\mathrm{Uc1}=\sum_{\mathrm{i}=1}^{\mathrm{n}} \frac{\left[\left(\mathrm{Ut} \mathrm{t}_{\mathrm{i}}+\mathrm{Uo}\right) \prod_{\mathbf{j}=1}^{\mathrm{i}-1}(\mathrm{Ij}, \mathrm{i})\right]}{\mathrm{n}}$

where,

Uc1 average combined utility for intradisciplinary coupling

$\mathrm{Ut}_{\mathrm{i}}$ utility value for a technology coupled to node/arc i

\footnotetext{
Formulated by Craig Johnson, Information Sciences, Inc., Denver CO under NASA contract NAS3-25266.
}

Uo utility of the nodal objective

Ij,i inheritance factor between the technology coupled to node arc $j$ and the similar technology coupled to node/arc i

n number of nodes and arcs constituting the scenario option

D2: The exact formulation ${ }^{* *}$ of the multidiscipline combined utility summation function is written as;

$U \mathbf{c 2}=\sum_{i=1}^{n} \frac{\left\{\left[\left(\sum_{i=1}^{k} U t_{I, i}\right)+U o\right]\left[\prod_{i=1, j=1}^{k}\left[G_{(i, j)}\right]\right]\right\}}{n}$

where,

Uc2 average combined utility for multidisciplinary coupling

$\mathrm{Ut}_{\mathrm{I}, \mathrm{i}} \quad$ utility value for a technology from discipline I coupled to node/arc i

Uo utility of the nodal objective

$G_{I(i, j)}$ synergy factor between technology i and technology $\mathrm{j}$ both coupled and operated at node I

k number of technology disciplines required at node/arc I

number of nodes and arcs constituting the scenario option 


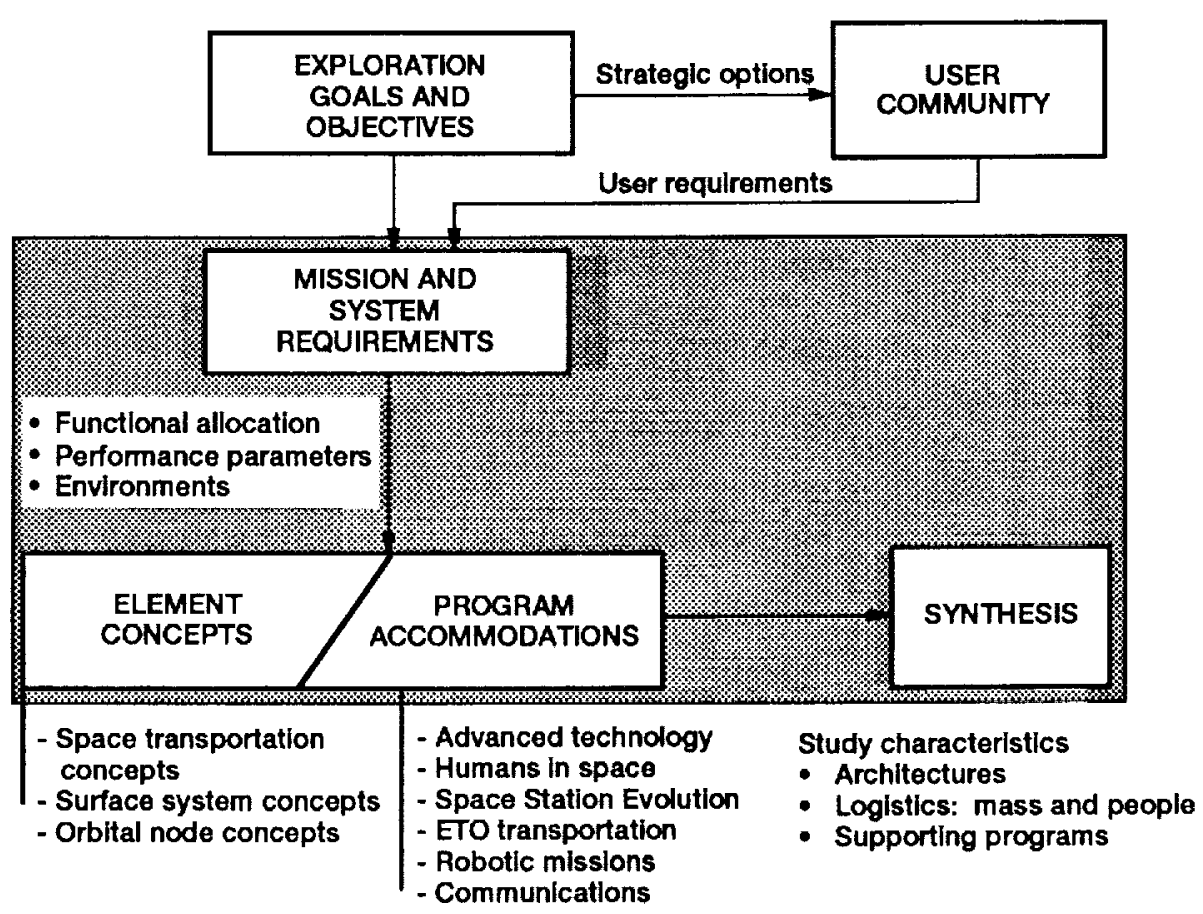

Shaded area covers all periods which could be supported to varying degrees by MisTec

Figure 1.-The study process.

(1)

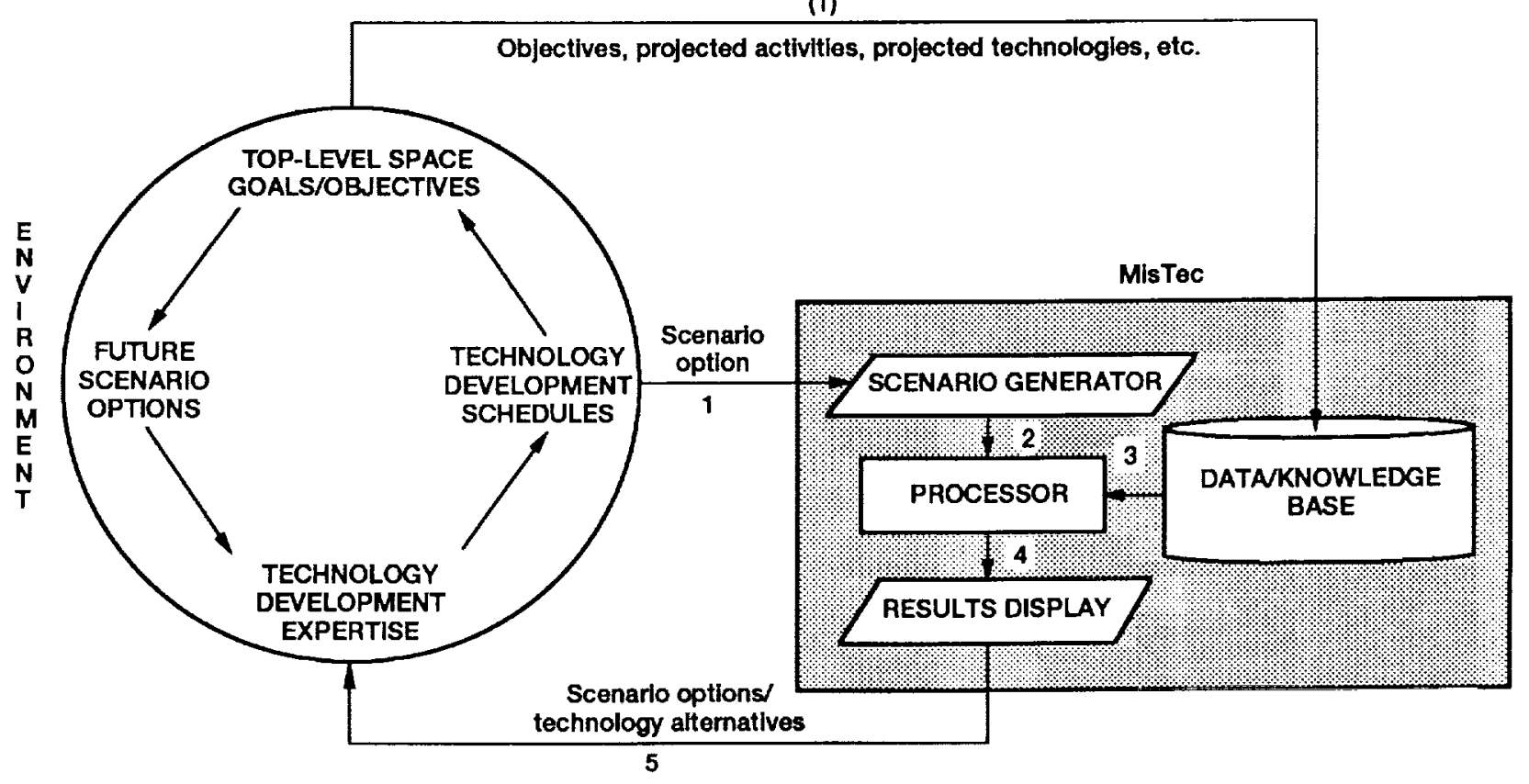

Figure 2.-Extemal and internal interfaces. 


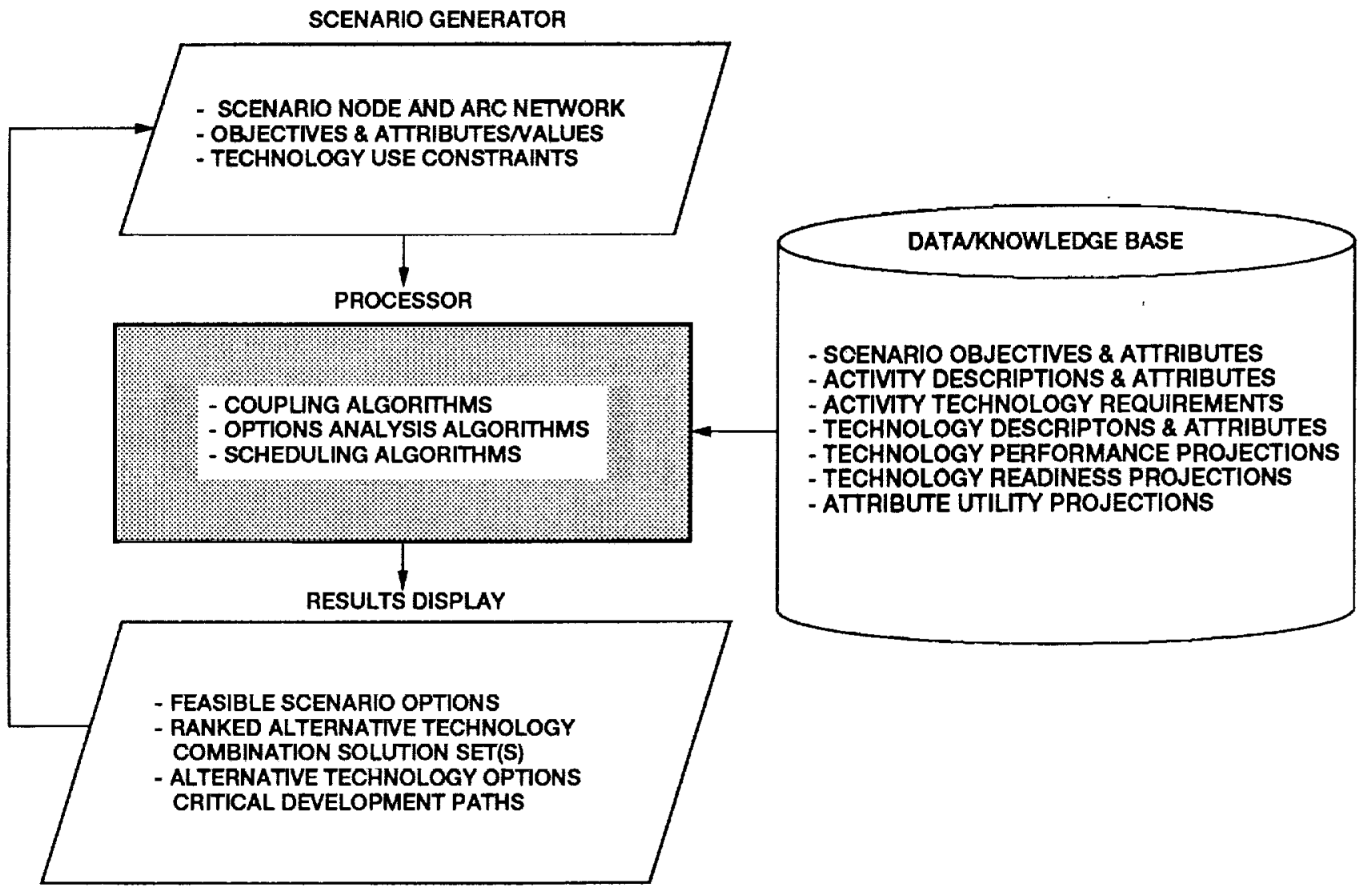

Figure 3.-MisTec internal functions and content. 


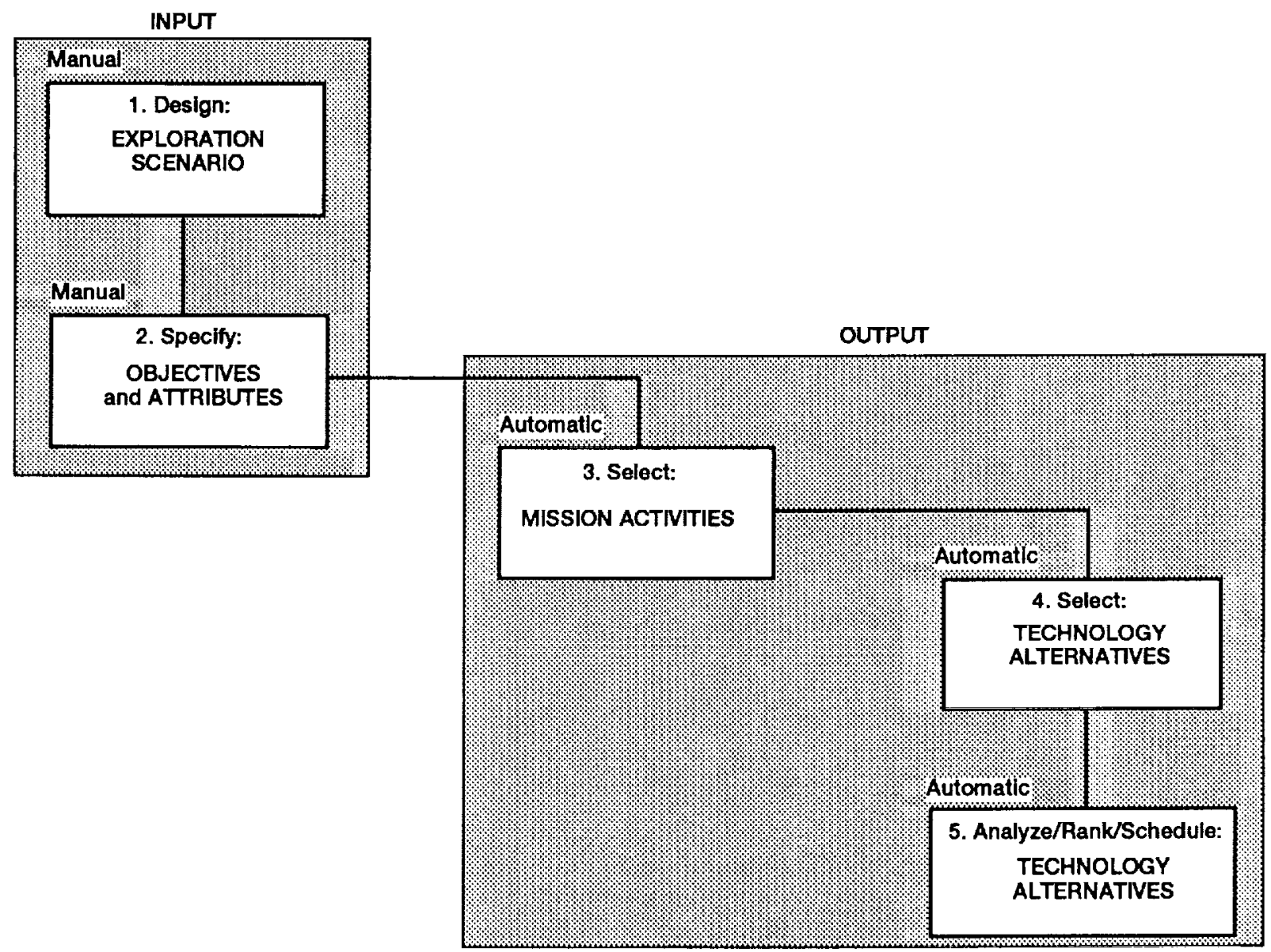

Figure 4.-MisTec operating procedure.

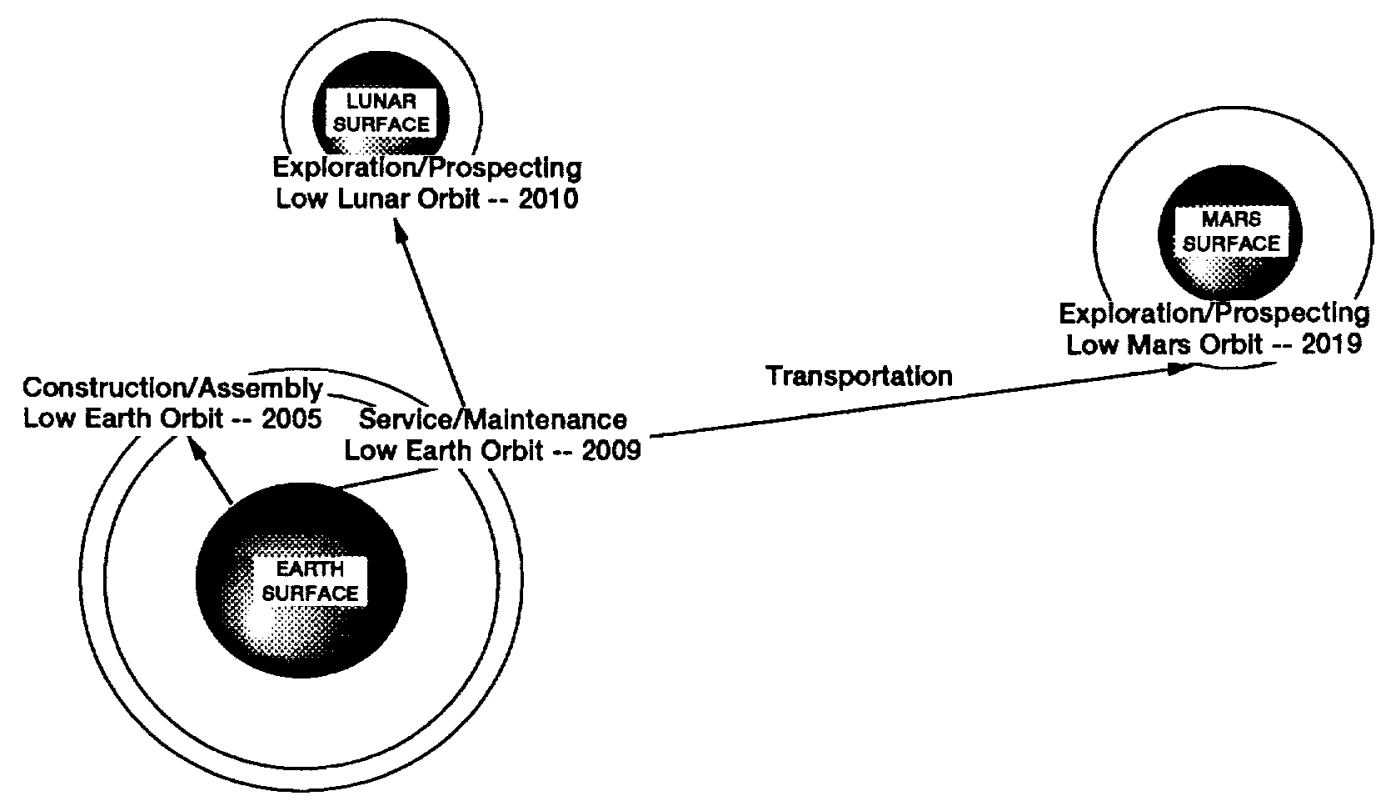

Figure 5.-Exploration scenarlo option, (example). 


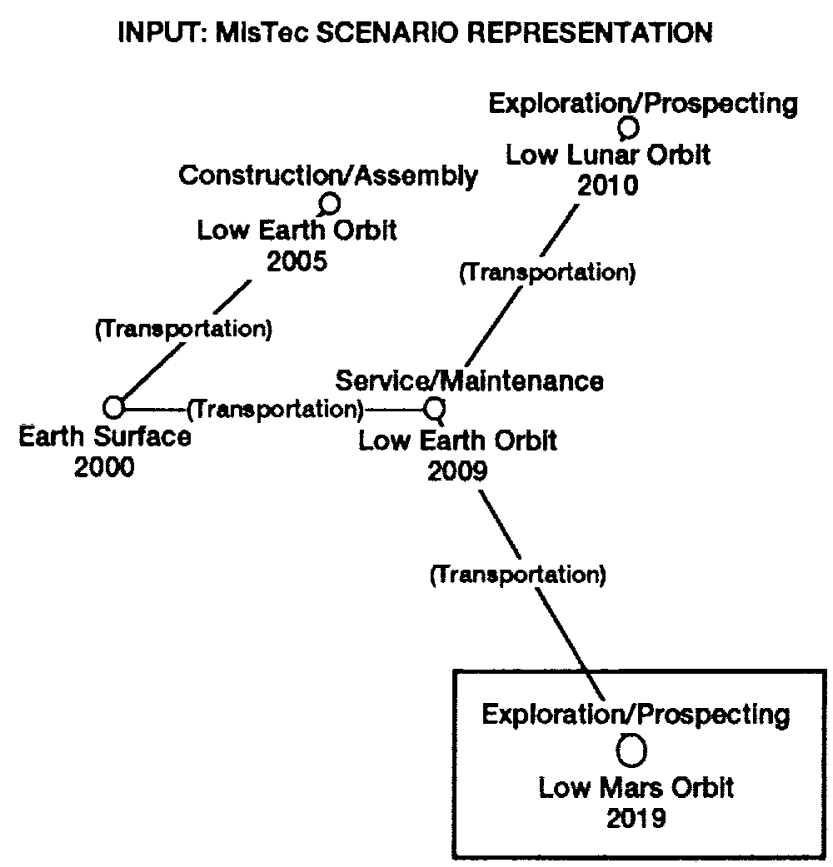

Figure 6.-Exploration scenario option, (example).

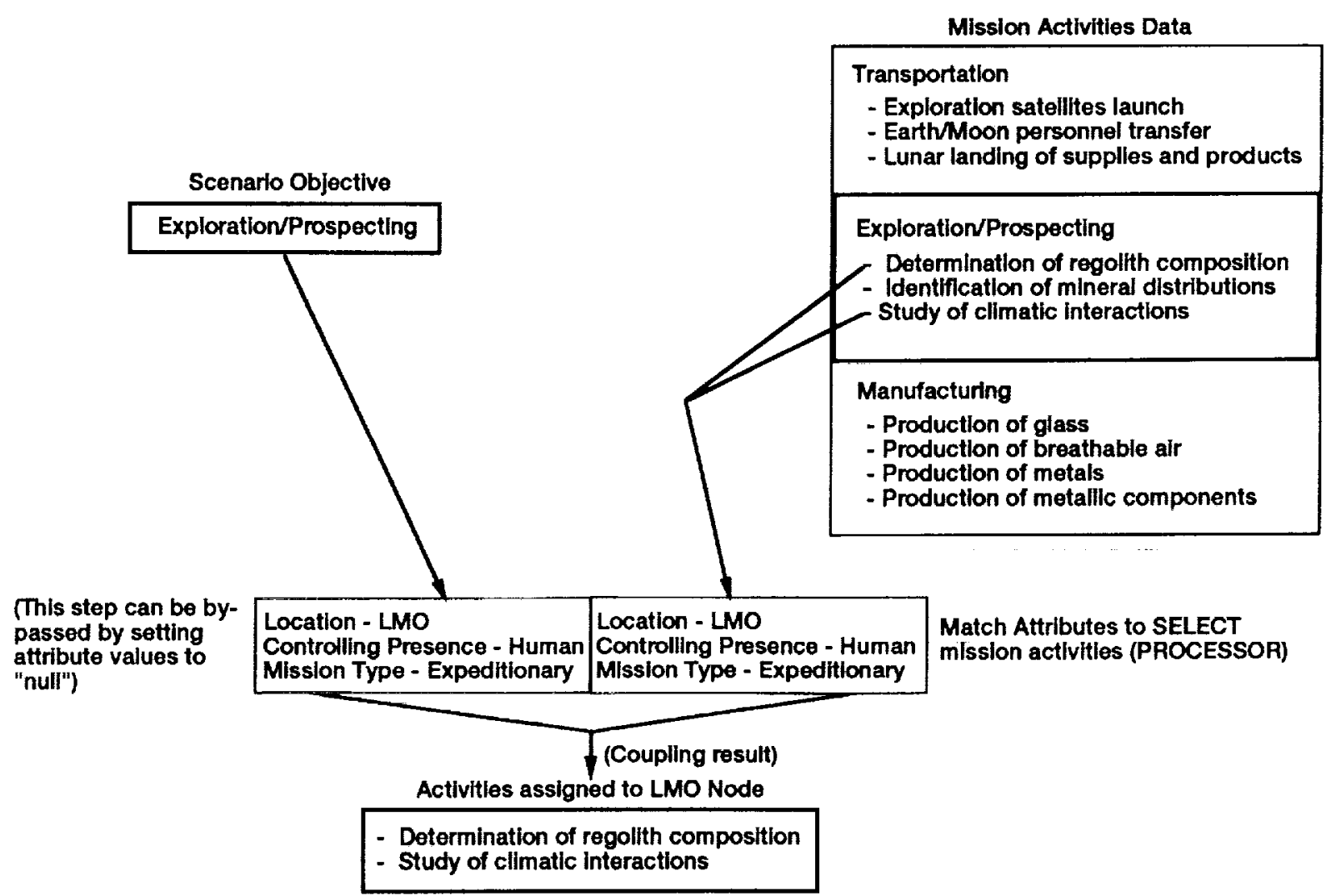

Figure 7.-Mission activity to node coupling (example of Low Mars Orbit (LMO) node). 


\section{Determine Nodal Power Requirement}

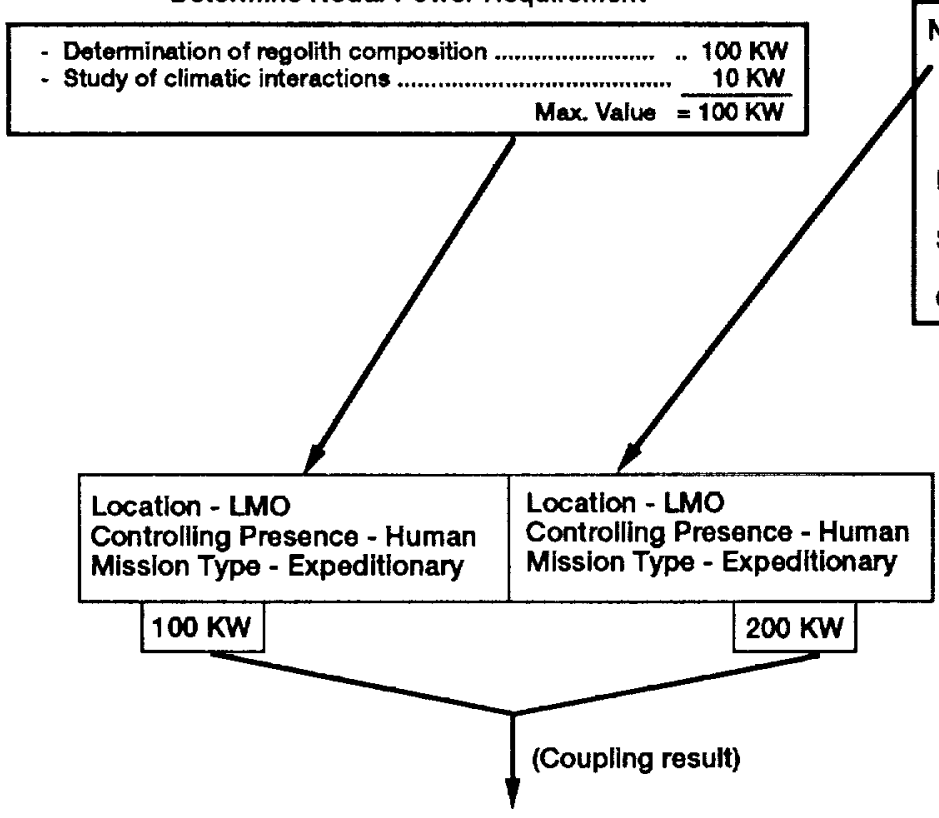

Technology assigned to LMO Node

Nuclear - Thermoelectric

Flgure 8.-Technology to node coupling (example for Low Mars Orbit (LMO) node).

OUTPUT: MISTEC COUPLING REPRESENTATION

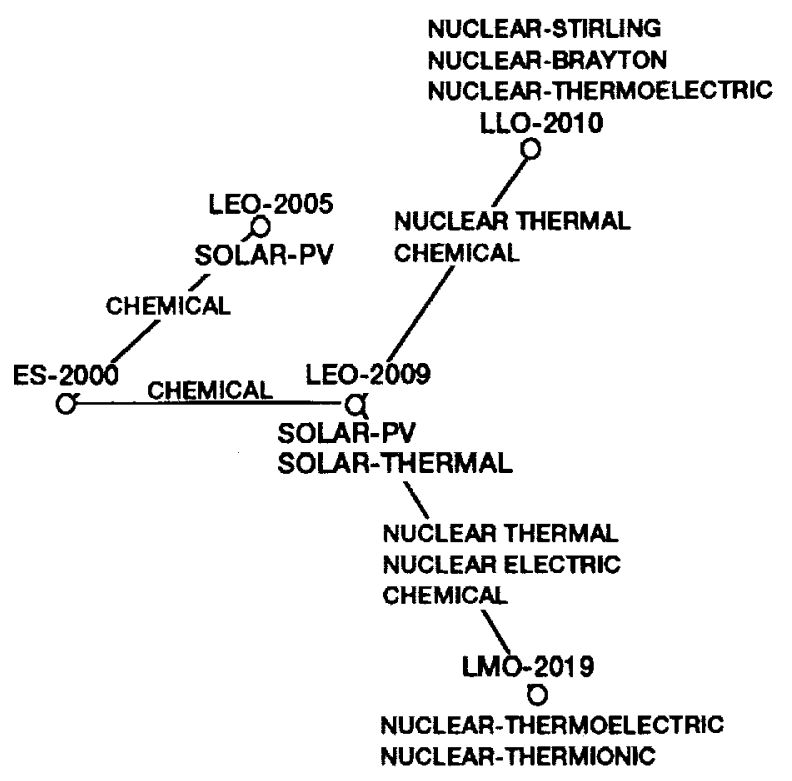

Figure 9.-Scenarlo with technology assignments, (example). 
OUTPUT: Options/ Atematives rankings

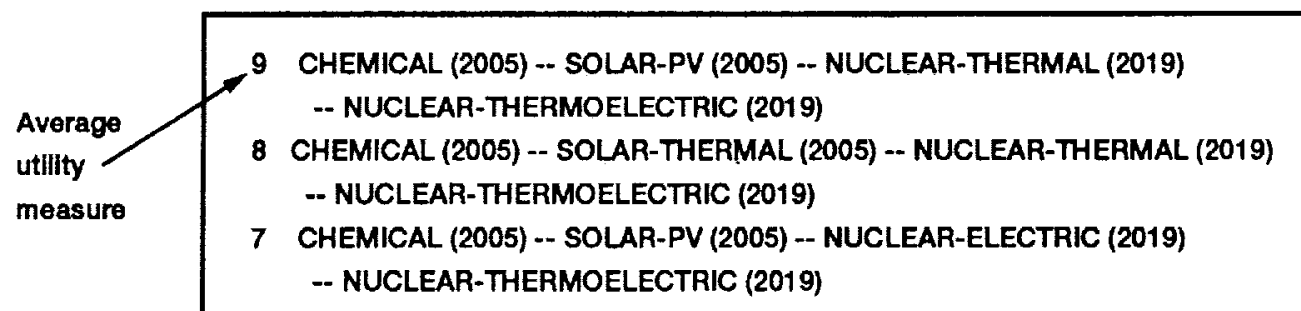

OUTPUT: Readiness Critical Path

SOLAR-PV

POWER LEVEL

Bace Environment

SPECIFIC POWER

Sace Envronment

EFFICIENCY

Sace Environment

...

NUCLEAR-THERMOELECTRIC

POWER LEVEL

Saco Environment

SPECIFC POWER

Sace Environment

sace

EFFICIENCY

Bace Environment

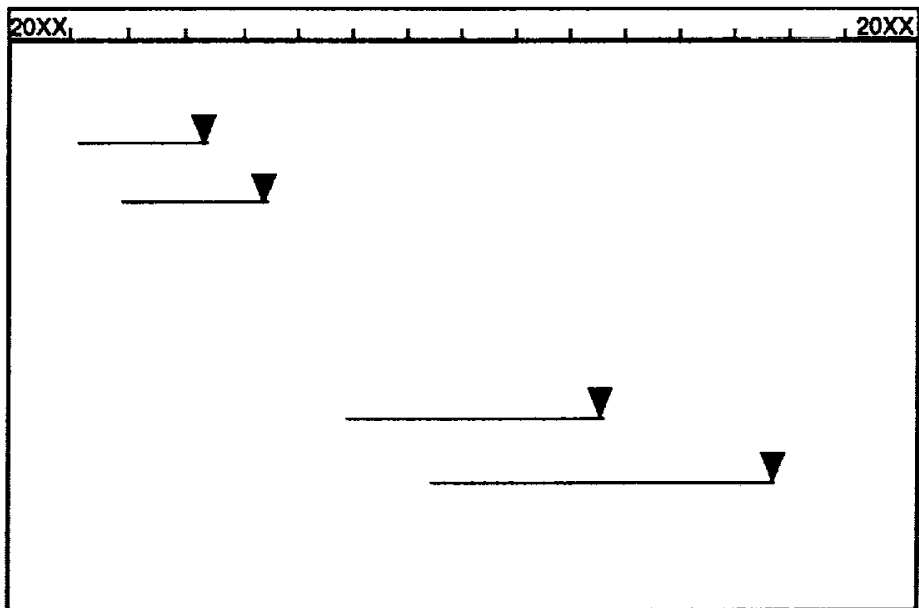

Figure 10.-Optlons/alternatives analysis, (example).

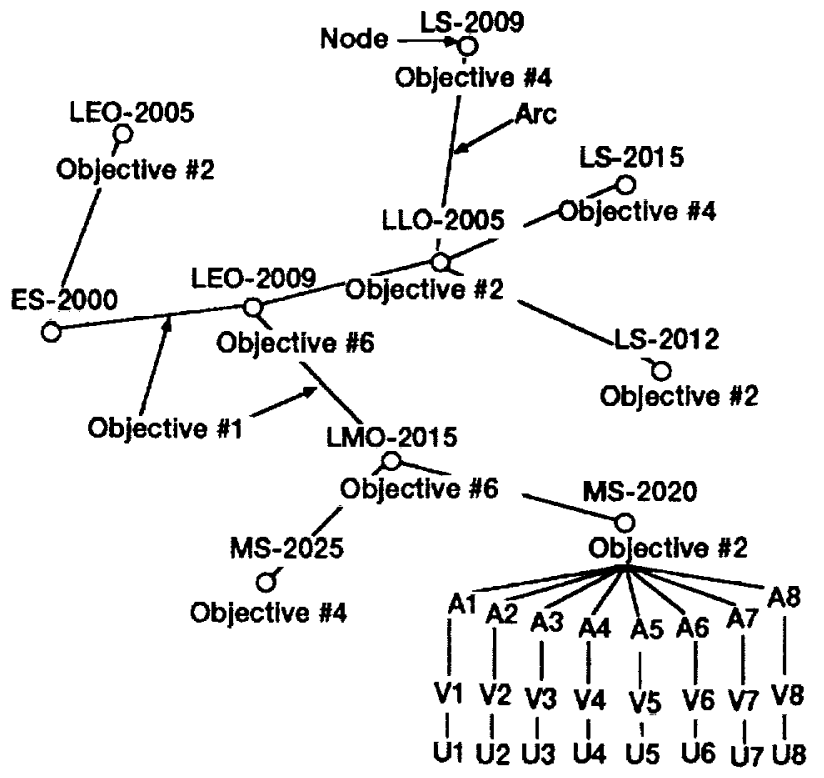

Figure b1.-General representation of a scenarlo optlon.
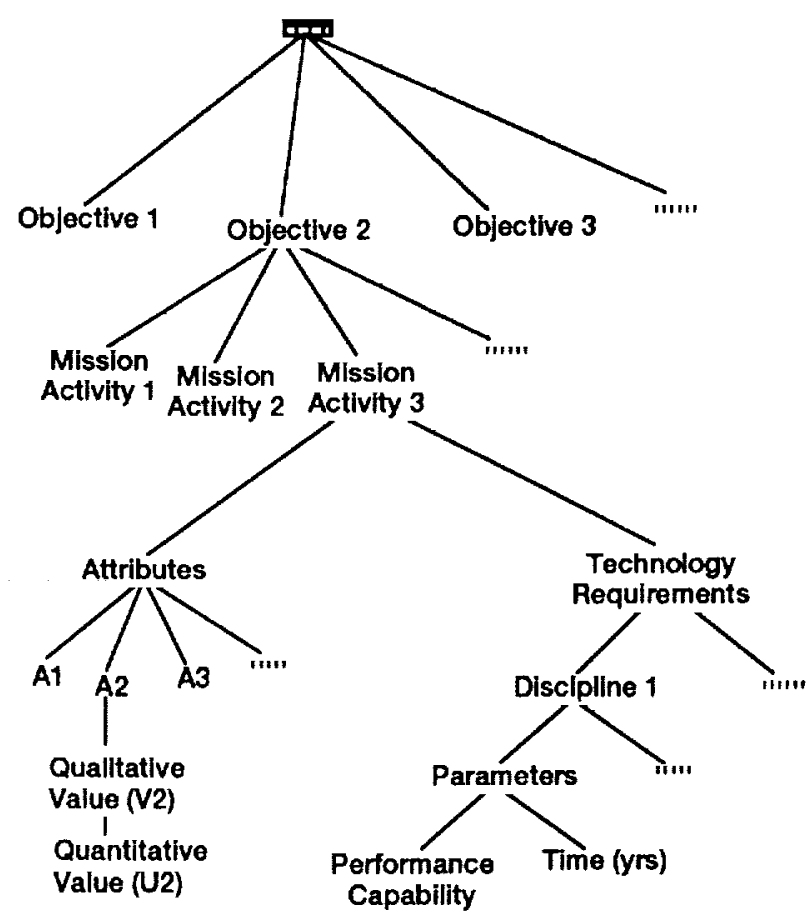

Figure c1.-Mission activity data tree: General structure. 


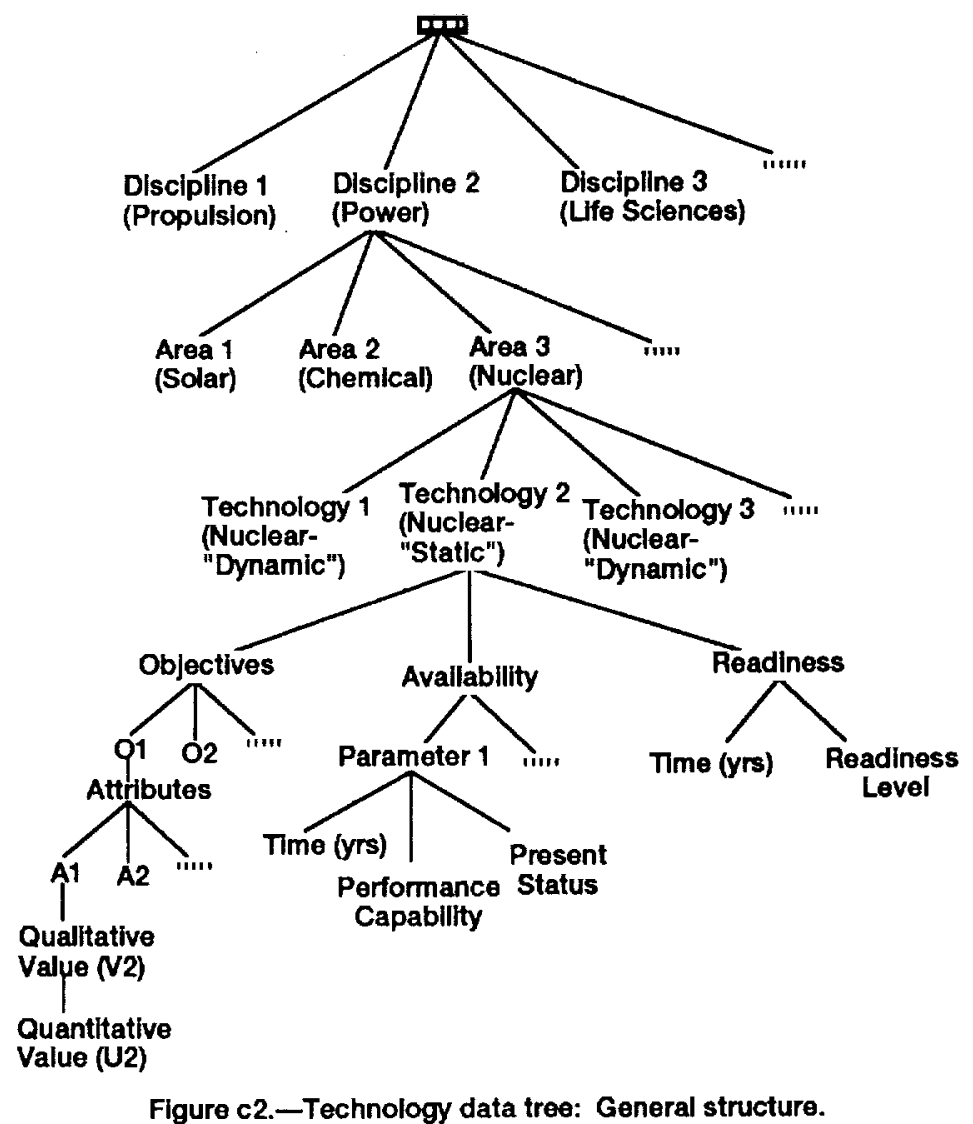

Figure c2.-Technology data tree: General structure. 
Fublic reporting burden for this collection of information is estimated to average 1 hour per response, Including the time lor reviewing linstructions, searching existing data sources, gathering and maintaining the data needed, and completing and reviewine the collection of information. Send comments reparding this burden estimate or any other aspect of this collection of information, Induding suggestions for reducing this burden, to Washington Headquarters Services, Directorate for information Operations and Reports, 1215 Jefferson Davia Highway, Suite 1204, Arlington, VA 22202-4302, and to the Olfice of Management and Budget, Paperwork Reduction Project (0704-0188), Washington, DC 20503.

\begin{tabular}{|l|l|l|}
\hline 1. AGENCY USE ONLY (Leave blank) & $\begin{array}{c}\text { 2. REPORT DATE } \\
\text { November } 1991\end{array}$ & $\begin{array}{r}\text { 3. REPORT TYPE AND DATES COVERED } \\
\text { Technical Memorandum }\end{array}$ \\
\hline
\end{tabular}

\section{TITLE AND SUBTITLE}

MisTec: A Software Application for Supporting Space Exploration Scenario Options and Technology Development Analysis and Planning

\section{AUTHOR(S)}

Gary A.P. Horsham

\section{FUNDING NUMBERS}

WU-594-81-21
7. PERFORMING ORGANIZATION NAME(S) AND ADDRESS(ES)

National Aeronautics and Space Administration

Lewis Research Center

Cleveland, Ohio 44135 - 3191
8. PERFORMING ORGANIZATION REPORT NUMBER

E-6529

\section{SPONSORING/MONITORING AGENCY NAMES(S) AND ADDRESS(ES)}

10. SPONSORING/MONITORING AGENCY REPORT NUMBER

National Aeronautics and Space Administration

Washington, D.C. 20546-0001

NASA TM - 105214

AIAA-92-1293

\section{SUPPLEMENTARY NOTES}

Prepared for the Space Programs and Technologies Conference sponsored by the American Institute of Aeronautics and Astronautics, Huntsville, Alabama, March 24-27, 1992.

12a. DISTRIBUTION/AVAILABILITY STATEMENT

12b. DISTRIBUTION CODE

Unclassified - Unlimited

Subject Categories 61 and 81

\section{ABSTRACT (Maximum 200 words)}

This paper presents the structure and composition of a new, emerging software application, which models and analyzes space exploration scenario options for feasibility based on technology development projections. The software application consists of four main components: a scenario generator for designing and inputting scenario options and constraints; a processor which performs algorithmic coupling and options analyses of mission activity requirements and technology capabilities; a results display which graphically and textually shows coupling and options analysis results; and, a data/knowledge base which contains information on a variety of mission activities and (power and propulsion) technology system capabilities. The general long-range study process used by NASA to support recent studies is briefly introduced to provide the primary basis for comparison for discussing the potential advantages to be gained from developing and applying this kind of application. The paper presents a hypothetical example of a scenario option to facilitate the best conceptual understanding of what the application is, how it works, or the operating methodology, and when it might be applied.

\section{SUBJECT TERMS}

Expert systems; Management information systems; Space exploration; Scenario; Architectures; Technologies; Analysis; Options; Strategy; Mission planning

\begin{tabular}{|c|c|}
\hline $\begin{array}{c}\text { 17. SECURITY CLASSIFICATION } \\
\text { OF REPORT } \\
\text { Unclassified }\end{array}$ & $\begin{array}{c}\text { 18. SECURITY CLASSIFICATION } \\
\text { OF THIS PAGE } \\
\text { Unclassified }\end{array}$ \\
\hline
\end{tabular}

19. SECURITY CLASSIFICATION OF ABSTRACT Unclassified
15. NUMBER OF PAGES 14

16. PRICE CODE $\mathrm{A03}$

20. LIMITATION OF ABSTRACT

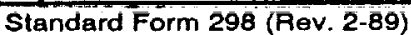
Prescribed by ANSI Std. Z39-1B 298-102 